\title{
Pharmacogenomics and pharmacogenetics: some cases in oncology
}

\author{
Rika Yuliwulandari ${ }^{1,2,3^{*}}$ \\ ${ }^{1}$ Department of Pharmacology, Faculty of Medicine, YARSI University, DKI \\ Jakarta 10510, Indonesia, ${ }^{2}$ Genomic Medicine Research Group, YARSI Research \\ Institute, YARSI University, DKI Jakarta 10510, Indonesia, ${ }^{3}$ The Indonesian \\ Pharmacogenomics Working Group
}

DOI: http://dx.doi.org/10.19106/JMedScieSup004804201627

\section{ABSTRACT}

Pharmacogenomics and pharmacogenetics play important roles in understanding how genetic variants influence drug efficacy and toxicity. In cancer cases, both efficacy and toxicity of therapeutic agents determine the improvement of patient's survival and quality of life. Effective treatment of cancer, one of the most deadly diseases in the world, is important to extend patient survival. Genetic variation influences the response of an individual to drug treatments in terms of efficacy and toxicity of the drugs. Understanding individual genetic variation is potential to achieve safer and more effective therapy, by tailoring drug options and dosage for each patient. In the context of cancer, tumors may have specific disease-defining mutations, but a patient's germline genetic variation will also affect drug response. Advance research technologies approach such as GWAS, next-generation sequencing technologies, statistical genetics analysis methods, and clinical trial designs have shown promises for the discovery of genetic variants associated to drug response in cancer. Understanding the molecular characteristics of both tumor and patient, and establishing their relations to therapy outcomes would be critical for the identification of predictive biomarkers as well as providing the basis for individualized treatments. Since cancer is also frequent in Indonesia, pharmacogenomics study in oncology and its implementation in the clinical practice become important. Broad collaboration at national and international level could foster the application of personalized medicine in oncology.

Keywords: pharmacogenomics, pharmacogenetics, germline, GWAS, nextgeneration sequencing 